\title{
Poster Abstract: A MAC Protocol for Medical Applications
}

\author{
Waltenegus Dargie and Jianjun Wen \\ Chair for Computer Networks, Technical University of Dresden, 01062 Dresden, Germany \\ Email:\{waltenegus.dargie, jianjun.wen\}@tu-dresden.de
}

\begin{abstract}
We propose a MAC protocol that supports the mobility of some nodes. An adaptive filter inside the protocol continuously evaluates the RSSI values of received acknowledgment packets and decides whether a mobile node should transfer a communication to a nearby relay node. This paper presents the design, implementation and evaluation of the MAC protocol.
\end{abstract}

Keywords-Handover, MAC protocol, mobility, sensor networks

\section{INTRODUCTION}

Wireless sensor networks that accommodate the free mobility of some of their nodes are obtaining increasing relevance in many areas, particularly, in medical instrumentation. For example, they have been proposed to supervise post-surgery rehabilitation after total hip and knee surgery (arterioplasty); to monitor and assist Parkinson Disease (PD) patients; and to diagnose sleep apnea outside a clinical setting [1].

Another application area is Biotelemetry. Traditionally, medical instrumentation involving Electrocardiography (ECG) and Electroencephalography (EEG) is used to monitor bioelectric potentials in the brain and the heart [2]. Whereas taking readings from these equipments in a clinical setting has several advantages, it has also some challenges. One of these refers to interference and transient effects from nearby operating electrical devices and the electrical power-system. The second challenge is that in a clinical setting the patient is at rest, bound to a bed or a medical couch. Biopotentials monitored under a resting position may not reflect the patient's actual condition in everyday life. Physically separating electrodes (deployed on the body of a patient) from the rest of the ECG/EEG (the processing subsystem) is considered to be the best solution for isolating the patient from the recording equipment and power lines [2]. It also enables the patient to freely move and stay active while brain and cardiac activities are monitored.

\section{A. Challenges}

One of the most formidable challenges in employing wireless sensor networks for medical applications is obtaining high quality data from the network. This may refer to accurately capturing temporal and spatial correlations. For example, the diagnosis of cardiac activities using ECG involves the determination of the so-called $P_{-}, Q R S$-, and $T$-wave complexes (see Fig. 1). The shape, amplitude and duration of these waves as well as their relative distance with respect to each other reveal normal and abnormal cardiac rhythms. Likewise, the absence, abnormal presence or relative distance of these waves

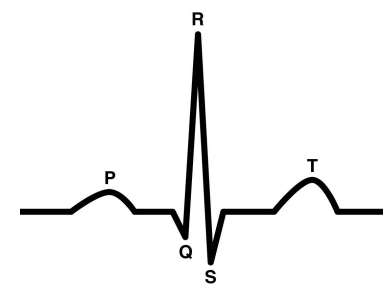

Fig. 1: PQRST-wave complexes of an electrocardiograph.

may indicate premature ventricular contraction, paroxysmal tachycardia, or atrial/ventricular fibrillation [2].

Therefore, wireless sensor networks dealing with mobile medical sensors should meet several critical requirements. For example, they should enable mobile nodes to establish a link with static relay nodes as quickly as possible (to reduce the packet transmission latency). Secondly, they should reduce the cost of frequent link disconnections and establishments. Thirdly, they should enable the transfer of a large amount of data once a link is established and before it should break. Fourthly, they should contribute to the optimization of the overall lifetime of the network.

\section{B. MAC Layer Support}

We propose a medium access control protocol that enables a seamless transfer of communication (handover) to address some of the issues raised in subsection I-A

1) Design: Our protocol is particularly suitable for a home environment where the mobile nodes are few in number. As far as medium access and low-power listening are concerned, it is similar to preamble-based protocols in wireless sensor networks [3], but it enables a mobile node to transmit packets

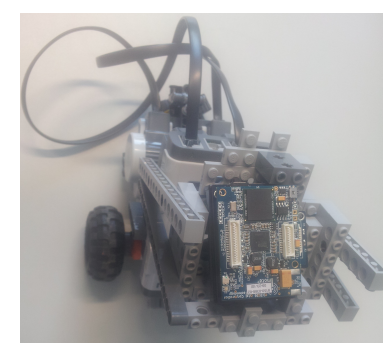

Fig. 2: A mobile Lego-robot carrying an IMote2 sensor node. 


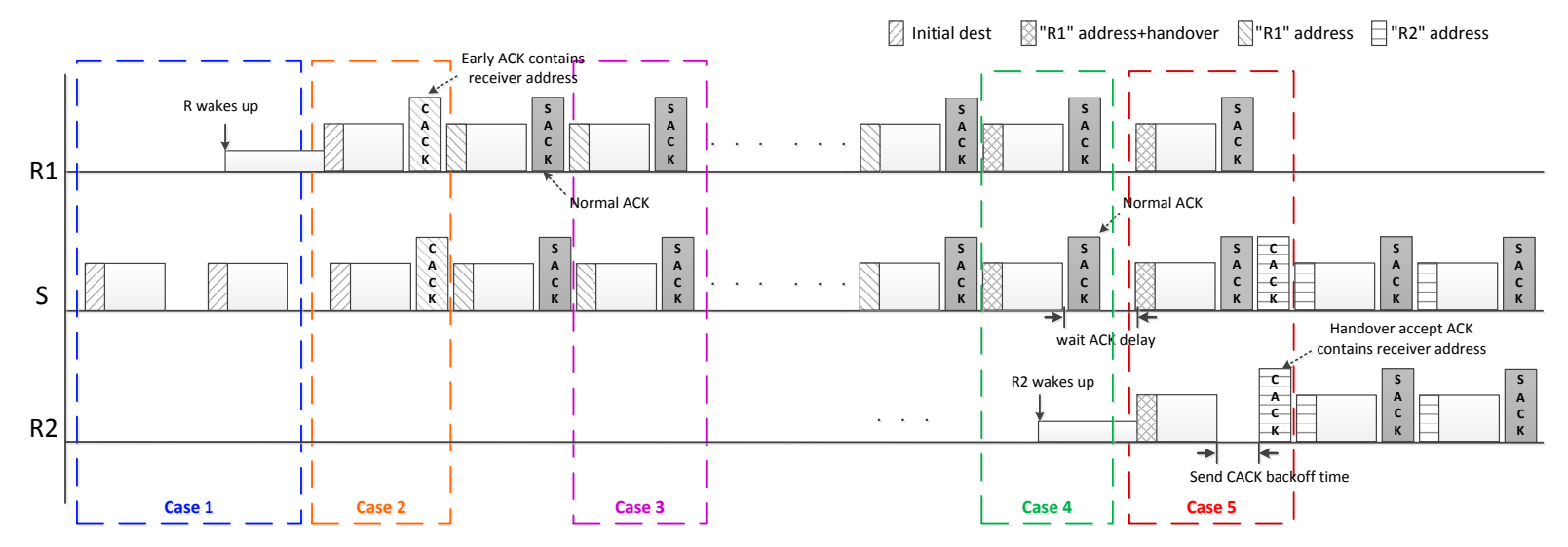

Fig. 3: A seamless handover support for a wireless sensor network with mobile nodes.

in burst once it wins the medium. This approach enhances channel utilization and high data delivery rate. Second, a Least Mean Square (LMS) adaptive filter inside the protocol continuously evaluates the RSSI values of received acknowledgment packets and initiates a seamless handover if it perceives a persisting deterioration in the link quality. To do this, we define three types of MAC addresses: a multicast address, a neighbor discovery address, and a unicast address.

In the beginning, a mobile node sends - after a successful contention for the medium - a preamble inside of which a multicast address is embedded. With this address it expresses an interest for a communication (Case 1 in Fig. 3). A nearby relay node responds to the preamble by embedding its own address in the acknowledgment packet (Case 2). A burst communication follows with each packet acknowledged by the relay node, but this time without embedding an address (Case 3 ). All other nodes in the vicinity refrain from contending to win the medium, until the burst transmission is completed. In the mean time, the LMS filter evaluates the RSSI values of the received packets and when it perceives a deterioration in the link quality (if the RSSI values drop below $-40 \mathrm{dBm}$ for our case), then it predicts the duration for which the RSSI will remain below this threshold. If this duration is longer than the time the mobile node requires to establish a new link, the protocol initiates a seamless handover (Case 4).

During a seamless handover, the mobile node replaces the unicast address with a neighbor discovery address but remains communicating with the original relay node. The neighbor discovery address enables both the surrounding relay nodes and the original relay node to understand the intention of the mobile node. With a slightly delayed response, the original relay node sends acknowledgment packets as usual while potential relay nodes can respond with their unique address to transfer communication to them (Case 5). If the LMS filter realizes that a better alternative relay node exists, then the mobile node addresses this relay node in that it communicates packets directly to this node by replacing the neighbor discovery MAC address with the relay's unicast address.

2) Implementation: We implemented a preamble-based MAC protocol for an IMote2 platform. We used TinyOS as our runtime environment and integrated the LMS filter, a duration

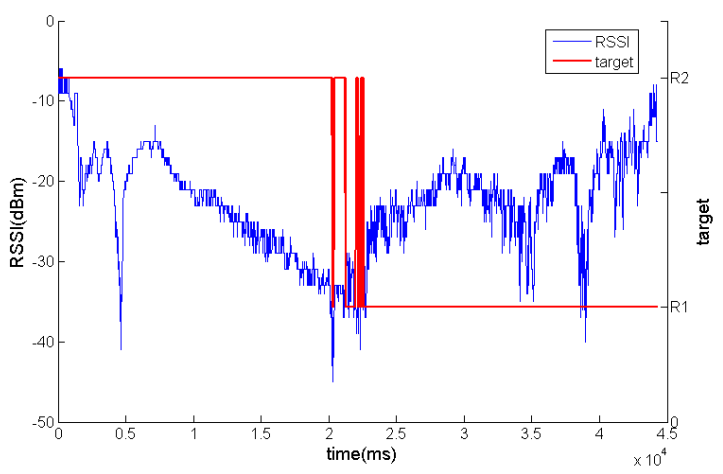

Fig. 4: A seamless handover taking place while a mobile Legorobot moves along a narrow corridor.

predictor, and a decision component in the low-power listening package of TinyOS. Our initial test has been encouraging. Fig. 2 shows a mobile Lego-robot carrying an IMote2 node and moving along a corridor. On its way, there were two relay nodes (R1 and R2) with which it can communicate. The distance between R1 and R2 was approximately $40 \mathrm{~m}$. Fig. 4 shows how the RSSI values of received ACK packets fluctuates as the robot moves and how the transfer of communication (the red line) changes as a result of the handover feature we implemented.

\section{CONCLUSION}

Initial experiment results show that a seamless handover feature at the MAC layer reduces single-hop latency and enables uninterrupted sensing. Additional experiments are required, however, to sufficiently quantify the advantages.

\section{REFERENCES}

[1] J. Ko, C. Lu, M. B. Srivastava, J. A. Stankovic, A. Terzis, and M. Welsh, "Wireless sensor networks for healthcare," Proceedings of the IEEE, vol. 98, no. 11, pp. 1947-1960, 2010.

[2] J. G. Webster, Medical Instrumentation Application and Design, 4th Edition. Wiley Publishing, 2009.

[3] W. Dargie and C. Poellabauer, Fundamentals of Wireless Sensor Networks: Theory and Practice. Wiley Publishing, 2010. 